\title{
The Simons Observatory: Overview of data acquisition, control, monitoring, and computer infrastructure
}

\author{
Brian J. Koopman ${ }^{\mathrm{a}}$, Jack Lashner ${ }^{\mathrm{b}}$, Lauren J. Saunders ${ }^{\mathrm{a}}$, Matthew Hasselfield ${ }^{\mathrm{c}}$, \\ Tanay Bhandarkar ${ }^{\mathrm{d}}$, Sanah Bhimani ${ }^{\mathrm{a}}$, Steve K. Choi ${ }^{\mathrm{e}, \mathrm{f}}$, Cody J. Duell ${ }^{\mathrm{f}}$, Nicholas Galitzki ${ }^{\mathrm{g}}$, \\ Kathleen Harrington ${ }^{\mathrm{h}}$, Adam D. Hincks ${ }^{\mathrm{i}}$, Shuay-Pwu Patty Ho ${ }^{\mathrm{j}}$, Laura Newburgh ${ }^{\mathrm{a}}$, \\ Christian L. Reichardt ${ }^{\mathrm{k}}$, Joseph Seibert ${ }^{\mathrm{g}}$, Jacob Spisak ${ }^{\mathrm{g}}$, Benjamin Westbrook ${ }^{1}$, Zhilei Xu ${ }^{\mathrm{d}, \mathrm{m}}$, \\ and Ningfeng $\mathrm{Zhu}^{\mathrm{d}}$ \\ ${ }^{a}$ Department of Physics, Yale University, New Haven, CT 06520, USA \\ ${ }^{b}$ University of Southern California, Los Angeles, CA 90007, USA \\ ${ }^{c}$ Center for Computational Astrophysics, Flatiron Institute, New York, NY 10010, USA \\ ${ }^{\mathrm{d}}$ Department of Physics and Astronomy, University of Pennsylvania, Philadelphia, PA 19104, \\ USA

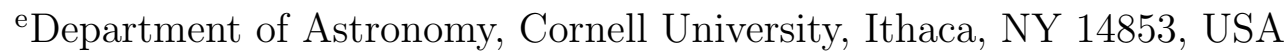 \\ ${ }^{\mathrm{f}}$ Department of Physics, Cornell University, Ithaca, NY 14853, USA \\ ${ }^{g}$ Department of Physics, University of California, San Diego, CA 92093-0424, USA \\ ${ }^{\mathrm{h}}$ Department of Astronomy and Astrophysics, University of Chicago, 5640 South Ellis Avenue, \\ Chicago, IL 60637, USA \\ ${ }^{i}$ Department of Astronomy \& Astrophysics, University of Toronto, 50 St. George St., Toronto \\ ON M5S 3H4, Canada \\ ${ }^{j}$ Department of Physics, Stanford University, CA 94305, USA \\ ${ }^{\mathrm{k}}$ School of Physics, University of Melbourne, Parkville, VIC 3010, Australia \\ ${ }^{1}$ Department of Physics, University of California, Berkeley, CA 94720, USA \\ ${ }^{\mathrm{m}}$ MIT Kavli Institute, Massachusetts Institute of Technology, Cambridge, MA 02139, USA
}

\begin{abstract}
The Simons Observatory (SO) is an upcoming polarized cosmic microwave background (CMB) survey experiment with three small-aperture telescopes and one large-aperture telescope that will observe from the Atacama Desert in Chile. In total, SO will field over 60,000 transition-edge sensor (TES) bolometers in six spectral bands centered between 27 and $280 \mathrm{GHz}$ to achieve the sensitivity necessary to measure or constrain numerous cosmological parameters, including the tensor-to-scalar ratio, effective number of relativistic species, and sum of the neutrino masses. The SO scientific goals require coordination and control of the hardware distributed among the four telescopes on site. To meet this need, we have designed and built an open-sourced platform for distributed system management, called the Observatory Control System (ocs). This control system interfaces with all subsystems including the telescope control units, the microwave multiplexing readout electronics, and the cryogenic thermometry. We have also developed a system for live monitoring of housekeeping data and alerting, both of which are critical for remote observation. We take advantage of existing open source projects, such as crossbar.io for RPC and PubSub, twisted for asynchronous events, Grafana for online remote monitoring, and docker for containerization. We provide an overview of the SO software and computer infrastructure, including the integration of SO-developed code with open source resources and lessons learned while testing at SO labs developing hardware systems as we prepare for deployment.
\end{abstract}

Keywords: Cosmic Microwave Background, Observatory Control System, Simons Observatory, control software, monitoring, data acquisition

Corresponding author: brian.koopman@yale.edu 


\section{INTRODUCTION}

The Simons Observatory ( $\mathrm{SO}$ ) is a new cosmic microwave background (CMB) experiment being constructed in the Atacama Desert in northern Chile. The observatory will consist of three small aperture telescopes (SATs) and one large aperture telescope (LAT). Spread among these telescopes will be over 60,000 cryogenic bolometers, measuring the temperature and polarization of the CMB from 27 to $280 \mathrm{GHz}$ in six frequency bands. These detectors will be read out using a microwave multiplexing ( $\mu \mathrm{MUX})$ architecture. ${ }^{1}$ The combination of small and large aperture telescopes was chosen to cover a wide range of science goals, which include measuring primordial tensor perturbations, further characterizing primordial scalar perturbations, constraining the sum of the neutrino masses and effective number of relativistic species, measuring the thermal and kinematic Sunyaev-Zel'dovich effects for a large sample of galaxy clusters, and measuring the duration of reionization. ${ }^{2,3}$

Orchestrating the control, data collection, and monitoring across all telescopes, their associated readout electronics, and the ancillary housekeeping (HK) electronics is a critical task for any observatory. Past CMB experiments have developed their own control codes or used those of similar experiments, for instance the General Control Program (GCP) developed initially on the Sunyaev-Zel'dovich Array and used across several other experiments including SPT, the Keck-array, BICEP2, and PolarBear. ${ }^{4,5}$ The Atacama Cosmology Telescope (ACT) developed its own remote control and monitoring infrastructure, which evolved over the years since the initial deployment in 2007. ${ }^{6-9}$ Generally, these control softwares have been closed source, and insufficient for the scale of hardware deployed on SO.

For the Simons Observatory we have designed and written a distributed control system we call the Observatory Control System (ocs). ocs is a modular system, designed to make it easy for any user to add additional hardware to the system. This design structure enables straightforward adaptation of existing code where similar hardware is used, and to extend the list of supported hardware for use on existing and new deployments of ocs.

In these proceedings we present an overview of ocs in Section 2, describing its components, how it is deployed, and its development process. Then, in Sections 3 and 4 we describe "Agents" and "Clients", the core components of ocs, and describe various ocs "Agents" and "Clients" that are written or planned. Next, in Section 5 we describe the live monitoring capabilities of ocs. In Section 6 we describe three different deployments; namely, a simple deployment, a deployment in one of the SO collaboration labs, and the planned deployment at the site in Chile, before concluding in Section 7. Appendix A contains Table 2 which lists all acronyms used throughout the text for reference.

\section{OCS OVERVIEW}

The ocs* is a distributed control system designed to coordinate data acquisition in astronomical observatories. A design goal has been ease of use in managing hardware operations and I/O tasks in distributed systems like those found at an observatory, where multiple processes must come together to record an observation. For a system to be easy to use, it should have a shallow learning curve, allow for users to add or modify components for their use cases, and have a simple installation procedure. The size of an ocs installation can be small, such as a single computer in a test lab, or complex such as a multi-computer system at the observatory. Throughout the rest of the paper, we refer to a single computer or CPU as a "node."

\subsection{Architecture}

The ocs has two primary components: ocs Agents and ocs Clients. ocs Agents are long-running software servers which interface with a hardware or software component to coordinate data acquisition. Each Agent contains a set of operations, referred to as "Tasks" and "Processes" that run for predefined or open-ended amounts of time, respectively. Tasks and Processes may be configured to run at Agent startup or may be initiated by a remote procedure call (RPC) over the network by an ocs Client. Information is passed among Agents on the network via a Publish/Subscribe (PubSub) pattern. Both RPC and PubSub are performed through a central Web Application Messaging Protocol (WAMP) router. The specific WAMP router implementation we have 


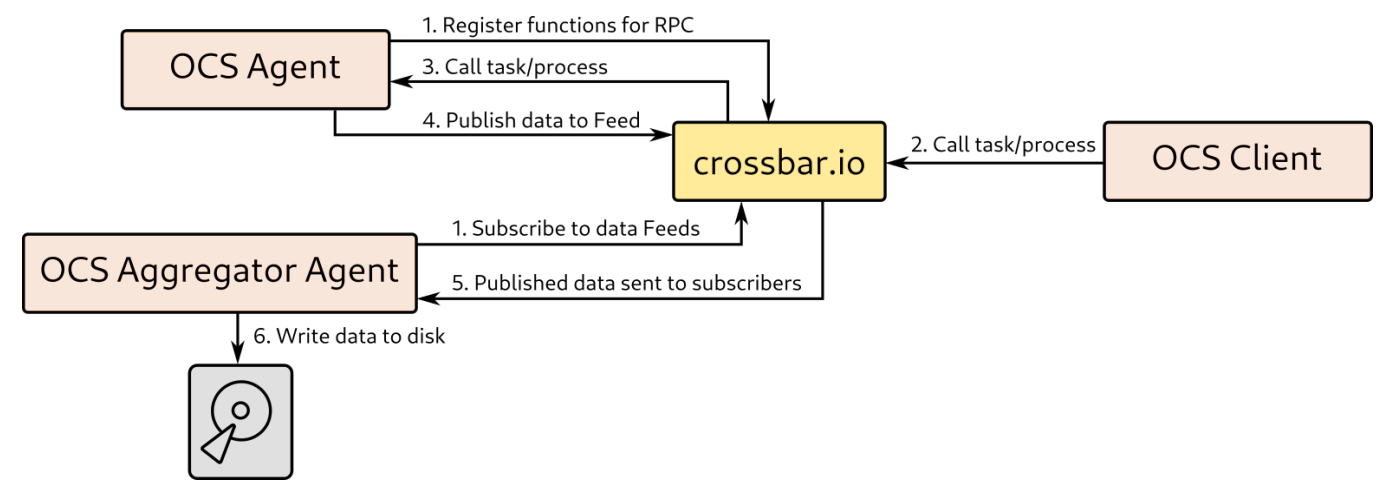

Figure 1. The interaction of two ocs Agents (a generic Agent, and the HK Aggregator Agent that writes data to disk), an ocs Client, and the crossbar server. Upon startup (1) the Agents both register their tasks and processes for remote calls and the ocs Aggregator Agent subscribes to all data Feeds, (2) an ocs Client makes a call to an agent task or process, (3) the crossbar server forwards this call to the Agent, (4) the Agent publishes data to an ocs Feed, (5) the HK Aggregator, a subscribed Agent, receives a copy of the data, and (6) writes the data to disk.

chosen is crossbar. io $^{\dagger}$ (referred to in this paper as just crossbar). A diagram showing the interaction between Agents, Clients, and the crossbar server is shown in Figure 1.

ocs Clients are scripts that orchestrate the actions of one or more ocs Agents on the network. Clients can take many forms, as crossbar supports several different programming languages. Most commonly, they are Python scripts run by the user on the command-line or JavaScript routines running in a web browser. Clients can also be a part of an Agent, commanding other Agents when needed. A good example of this is the Observation Sequencer described in Section 3.3.

Clients can be run anywhere on the network that has access to the crossbar server, such as a dedicated computer at the site or a user's laptop. Agents can be run directly on bare metal; however more commonly they will be run within Docker containers for ease of setup, use, and monitoring, as described in Section 2.4.

\subsection{Dependencies}

We leverage several open source tools in ocs, which we describe in this section.

crossbar is an open source implementation of the Web Application Messaging Protocol (WAMP) used for distributed applications. WAMP $\ddagger$ is a websockets subprotocol that provides two application messaging patterns, RPC and PubSub. Developed by Crossbar.io (the company), crossbar.io (the software) is accompanied by the Autobahn libraries which provide open source WAMP implementations in several programming languages. This was appealing when selecting a mechanism for RPC and PubSub, as it allowed support for Python, JavaScript, and, if needed, $\mathrm{C}++$. These Autobahn libraries provide the base components which allow one to write WAMP "application components" that we use to run the ocs Agent code, described in more detail in Section 3.

The WAMP application components are necessarily asynchronous. Autobahn offers the choice of either of the asyncio or twisted libraries for implementing the asynchronous networking framework; we use the latter.

We selected the SPT3G software (spt3g_software) framework ${ }^{\S}$ for the ocs data storage format. ${ }^{10}$ The code is written in $\mathrm{C}++$ with a light Python layer. The internal structure of the files consist of "Frames", which are processed through "Pipelines" by "Modules" one at a time. We have built a new library, so3g, ${ }^{\mathbb{I}}$ which operates on these standard G3 frames, taking advantage of the structure of the underlying spt3g_software file format, while providing helper methods for loading data without users needing to consider the frame structure.

\footnotetext{
*https://github.com/simonsobs/ocs

${ }^{\dagger}$ https://crossbar.io

${ }^{\ddagger}$ https: //wamp-proto.org/

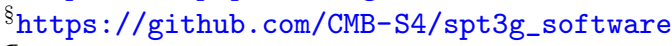

Ihttps://github.com/simonsobs/so3g
} 
Housekeeping (HK) data are collected and written to SPT3G files (or .g3 files) by the HK Aggregator Agent, where frame length and file length are configurable parameters. Detector data are collected by a G3 pipeline called the "SMuRF Streamer" and are sent over the network to the SMuRF Recorder Agent, where they are written to .g3 file on disk. For more information on these Agents see Section 3.

\subsection{Configuration}

The Agents and connection information for the WAMP router are defined within an ocs Site Configuration File (SCF). This is a simple YAML file, containing the address for the WAMP router, some internal WAMP address information, and a list of each Agent, grouped by which host they will run on. Each Agent is defined by its "agent-class", a unique name given to each type of Agent and an "instance-id", a unique name given to a particular instance of a type of Agent (the latter is necessary because many copies of a single Agent may be running on the network, say for interfacing with multiple identical pieces of hardware). Command-line arguments to further configure Agents are passed to the Agent as a list.

The SCF, together with the Docker Compose configuration files that define the Docker containers in which individual ocs Agents run (see Sec. 2.4), completely define the ocs network. This makes the system incredibly easy to migrate to a different machine, or to restore to a machine that might need to be replaced due to a hardware failure or any other reason. In addition, an expert is often able to identify misconfiguration by viewing just these two configuration files.

If an Agent is deployed outside of the Docker framework, the SCF defines the paths to the Agent's code. These paths may be split into different repositories. The ocs repository provides core Agents critical to the functionality of ocs, while Agents specific to the Simons Observatory hardware are kept in a separate repository called socs (the Simons Observatory Control System)."

\subsection{Docker}

We use Docker extensively in the deployment of ocs within the Simons Observatory. It allows for the packaging of software, libraries, and configuration files for each Agent into shareable Docker images. These isolated, reproducible environments for each Agent have been valuable in the deployment of ocs in labs throughout the SO collaboration. Agents are built into images and published to Docker Hub, ${ }^{* *}$ an online resource for hosting and downloading public Docker images. This is done automatically by the Continuous Integration pipelines described in Section 2.6.

Docker Compose is used to start up the multi-container environment needed for ocs. Long-running services such as the crossbar server, InfluxDB, and Grafana are managed separately, and configured to start at boot. This allows for the ocs stack to be restarted in a straightforward manner without affecting these third party services. Orchestration tools such as Docker Swarm, for managing docker containers across the network are still being explored, though we note that Swarm does not support device connections like those needed for USB hardware such as the Lakeshore 240 (a thermometry readout system).

\subsection{Open Source, Documentation, and User Contributions}

Clear documentation is vital for adoption of any software package. Since the core of ocs is written in Python we make use of the Sphinx tool ${ }^{\dagger \dagger}$ for creating our documentation, which is freely hosted on Read the Docs, ${ }^{\ddagger \ddagger}$ an automatic building, versioning, and hosting platform for open source project documentation. Each commit to the ocs repositories triggers a new build of the documentation which is automatically updated on the Read the Docs website, and publicly viewable.

Documentation is split into several sections, including a User guide that provides a walk-through of the setup of an ocs network, the Agent reference detailing what each ocs Agent does and how to use and configure them, and a Developer guide describing in more detail how core components of ocs work and how users can develop

\footnotetext{
"https://github.com/simonsobs/socs

**https://hub.docker.com/u/simonsobs

${ }^{\dagger \dagger}$ https://www.sphinx-doc.org/en/master/

${ }^{\ddagger}$ https://readthedocs.org/
} 
their own Agents and Clients. This thorough documentation has been key to the development and use of ocs, including among the test labs within the Simons Observatory.

The core of ocs and the first Agents were initially developed by a small team of developers. This allowed for rapid development of the structure of ocs and demonstration of the ability to interface with specialized hardware in the lab. The first example use case was temperature readout and control of a ${ }^{3} \mathrm{He} /{ }^{4} \mathrm{He}$ dilution refrigerator, primarily through the associated low noise resistance bridges and cryogenic temperature modules produced by Lakeshore Cryotronics.*

As test labs deployed more and more hardware during the design and building phase of SO they began using ocs installations to facilitate hardware testing. It is much easier to develop and debug new Agents when one has direct access to the requisite hardware. Thus, researchers in multiple labs developed new Agents for their specific hardware by modeling their work on existing Agents with the help of the ocs documentation. This practice has made the integration of hardware into the experiment relatively straightforward. It has also been valuable for developing ocs since we have had several testing environments to work out bugs in the core ocs code.

\subsection{Pipeline}

The development of ocs has benefited from the use of Continuous Integration (CI), namely, the practice of frequently integrating code to a shared repository, triggering the automated building and testing of code changes. We initially used Travis CI as our CI platform, but recently migrated to GitHub Actions, which integrates nicely with GitHub and allows simpler control over workflows triggering on specific events. The initial motivation was to automate the building of updated Docker images for the Agents upon new commits. Before the use of continuous integration, these images would need to be built by hand and pushed to a Docker registry for distribution, an easy step to forget during development.

There are several workflows defined with the ocs repositories. The first focuses on testing. ocs has a set of unit tests written to be run with pytest. The workflow will build the Docker images for each ocs component and run the unit tests within an ocs container. Code coverage is reported, extracted from the container, and published to the Coveralls platform. ${ }^{\dagger}$ Finally the documentation is built within a container to test for any failures in that process, as we have found it easy to break Sphinx builds on Read the Docs, especially when a new python module dependency is added. This workflow is valuable as it catches any bugs introduced by new changes that might break the unit tests. It is run on any commit and pull request, providing rapid feedback to contributing developers.

The other workflows focus on the building and pushing of Docker images to Docker Hub. We distinguish between the stable releases of ocs and the development branch of ocs. Development images are built and pushed on merge commits to the "develop" branch. These have descriptive tags and allow for deployment of images under testing before the next official versioned release. Official releases of ocs trigger a separate workflow which also builds Docker images with succinct tags corresponding to the release version, as well as the common "latest" tag for Docker images. In both workflows these images are pushed to Docker Hub. Stable images are only built on the main ocs branch, which is branch-protected and only merged to on scheduled releases.

\section{OCS AGENTS}

The ocs Agent is one of two major components of ocs. Each ocs Agent is a subclass of the Autobahn ApplicationSession class. Connection and disconnection events that occur during the application life cycle are overridden to provide ocs functionality. On startup, these handle the registration of tasks and processes for remote calls from ocs Clients, the startup of any configured task or process, and the startup of the Agent heartbeat, a signal of Agent health. On shutdown they handle the stopping of any running tasks or processes, and shut down of the Agent. If an Agent for some reason becomes disconnected from the crossbar server, it attempts to reconnect for a short period of time before shutting down altogether. This allows for brief outages of the crossbar server, say, for instance, if it needs to be restarted (though in practice the need for this is rare).

\footnotetext{
*https://www. lakeshore.com

†https://coveralls.io/
} 
The OCSAgent class provides useful wrappers for PubSub and for providing the Agent operation commands. Each agent that will publish data must first register the communication channel, which we call the ocs Feed, a class which handles the WAMP address space and the buffering and publication of data submitted to it by an Agent. Once a Feed is registered, the Agent can publish data to it in "blocks", each of which contains co-sampled data, whether for a single timestamp or a longer duration of time. The block structure is not allowed to change after construction. Its structure, published to a Feed, is a simple Python dictionary. These data are serialized before being sent over the network. Prior to publication, the Feed performs some verification of the message data structure. This check at the Feed level can be useful for debugging when developing an Agent.

Any agent, or supporting client, can subscribe to an ocs Feed. Agents that aggregate all data from other Agents, like the HK Aggregator or InfluxDB Publisher Agent, subscribe to all ocs Feeds that are marked for recording to disk.

Tasks and Processes made available for remote call in an Agent have a simple operation control API that provides four possible actions: "start", "status", "wait", and "abort" or "stop" (for Tasks and Processes, respectively). "Start" will request that a task or process be run, passing any parameters needed for the operation. "Wait" will block until the operation has exited or a timeout has elapsed. "Status" returns the current status of the operation. Possible values are "starting", "running", "stopping", and "done". The returned object can also include informative data, such as the most recent data collected by the Agent in a structure we call the operation session data. The "abort" or "stop" methods will abort the task or process. These operation control actions are called by ocs Clients.

In the remainder of this Section we present details on several of the major ocs Agents, including those used for pointing the telescope, commanding the readout electronics, writing data to disk, and orchestrating observations. We also present a summary of all other supporting Agents.

\subsection{Antenna Control Unit}

The Antenna Control Unit (ACU) is an industrial PC used to control the motion of a single telescope platform. The Simons Observatory will use one ACU per telescope, provided by Vertex Antennentechnik, ${ }^{\ddagger}$ the company that has been commissioned to build the telescope platforms. Vertex Antennentechnik additionally provides software on the ACUs that provides an API for controlling the telescope platform, allowing users to switch between the operational modes required for observations. The ACU also publishes two data streams to ports on a computer provided by SO: a $200 \mathrm{~Hz}$ UDP stream containing time stamps and encoder values, and a $5 \mathrm{~Hz}$ TCP stream containing those same values in addition to monitors for errors and faults.

The ACU ocs Agent publishes the data received from each of these streams to an ocs Feed for recording to file and for viewing in the live monitor. The Agent also provides Operations for commanding the telescope. The functioning of the incoming data monitors has been verified during factory acceptance testing for the first commissioned SAT at the Vertex Antennentechnik facility in Germany; testing of some tasks remains ongoing.

\subsection{SMuRF Agents}

The Simons Observatory will read out its detector array via superconducting quantum interference device multiplexing ( $\mu \mathrm{MUX}$ ) with the SLAC Microresonator Radio Frequency (SMuRF) warm electronics system. ${ }^{1,11}$ Communication with the SMuRF system is facilitated by the rogue ${ }^{\S} \mathrm{C}++$ software library. rogue implements low-level communication with the SMuRF FPGA, providing register access and asynchronous data streaming. It includes an Experimental Physics and Industrial Control System (EPICS) server that allows register reading and writing operations to be executed from high-level clients, such as the Pysmurf library, a Python library provided by SLAC for interfacing with the SMuRF systems. I

A dedicated SMuRF server is used to run rogue, Pysmurf, and related ocs Agents, and to temporarily store auxiliary data before it is copied to a more permanent storage node where it is archived along with the ocs housekeeping data. For detector data acquisition, we are using a custom rogue plugin that collects detector

\footnotetext{
${ }^{\ddagger}$ https://www . vertexant.com/

$\S_{\text {https://github.com/slaclab/rogue }}$

$\mathbb{I}_{\text {https: //github.com/slaclab/pysmurf }}$
} 
data and relevant metadata through the asynchronous streaming interface. Data are grouped into chunks of about one second and packaged into serializable frames using the SPT3G data format. Frames are sent over the network to the storage node where they are received by the SMuRF Recorder ocs Agent that writes them to disk.

Control of the SMuRF system and high-level analysis is managed with the Pysmurf library, which utilizes the EPICS interface to control the hardware. In development, Pysmurf is primarily controlled interactively through an iPython interface or a Jupyter notebook. Once common procedures have been established, functions can be run through ocs using a Pysmurf Controller agent that manages the execution of Pysmurf scripts.

Pysmurf generates important companion data such as plots and summaries that describe detector parameters and operating behavior. This companion data must also be archived along with the detector data, which is done through the use of the Pysmurf Monitor and Pysmurf Archiver ocs agents. Pysmurf passes the auxiliary file metadata to the Pysmurf Monitor Agent running on the server, which then writes the metadata to a database on the storage node. The Pysmurf Archiver runs on the storage node and monitors the database for new metadata entries and then copies the corresponding files over to be archived along with the detector and housekeeping data.

\subsection{Observation Sequencer}

The Observation Sequencer Agent orchestrates observatory systems to perform observations. In this sense it is also an ocs Client, issuing commands to other Agents on the ocs network. The sequencer requests the survey plan from a separate program and executes a series of commands, e.g. to the various SMuRF Agents to ready the detectors, to the ACU Agent to point the telescope.

The Sequencer will record its performed actions in a database to support downstream monitoring. This will be used to flag errors during data acquisition, confirm the data for a given observation were acquired, associate the data on disk with the given survey plan, and group data files and calibration operations together. The Sequencer is currently in a development phase.

\subsection{Additional Hardware Agents}

Table 1 shows a summary of other ocs Agents that have been written or are under development for use on the Simons Observatory. These Agents are easily shared among other ocs users and several are user-contributed. Beyond the Agents listed here are more to be developed, including Agents to interface with Fourier Transform Spectrometers, polarization grid calibrators, cooling loops, additional power supplies, and more.

\section{OCS CLIENTS}

ocs Clients are the programs that command single or multi-agent sites, orchestrating device operation and data collection across the observatory. Most commonly these will be Python scripts, JavaScript embedded in a users web browser, or subroutines within ocs Agents. Clients can connect to the crossbar server either via websockets, for instance, using the wampy python package, or via HTTP or HTTPS. In order for clients to support subscription, they must connect via websockets and not the HTTP bridge.

Within ocs there are two methods for writing a Python based Client: the Basic Client, in which the user must define each operation they wish to call, and the Matched Client, which performs this definition automatically, matching operation calls to their defined task or process names. By default Basic Clients connect via websockets, while MatchedClients connect via HTTP, though both backends are supported in either case. A Matched Client, communicating over HTTP is perhaps the simplest Client for getting started quickly, and for simple interactions with running Agents. Clients can be run from anywhere on the network where the crossbar server is run, allowing for users to send commands from their laptops as simply as from on-site computing infrastructure. Javascript clients are discussed in Sec. 4.1. 


\subsection{OCSWeb}

OCSWeb is an ensemble of ocs Clients implemented in JavaScript and rendered as a GUI in a web browser. Two screenshots are shown in Figure 2. The elements of OCSWeb are a JavaScript library, HTML and CSS files to present a basic interface and styling, and a variety of Agent-specific JavaScript modules to produce tailored interfaces.

For basic functionality, the browser must be able to connect to crossbar through the websocket interface. The main page of the interface presents a configuration box and a list of active ocs Agents. Users may interactively select different Agents and browse through the Tasks, Processes and Feeds presented by each one, including the latest session data and log information. The main page does not permit any detailed control but is useful for basic health monitoring, debugging and development purposes.

Control panels that are tailored to specific Agent types can be launched from the main interface, with each appearing in a new tab of the main window. The control panels are rendered according to JavaScript code developed specifically for that Agent class. As is the case for the Agent code itself, the creation of Agent-specific panels is largely left in the hands of developers working with those Agents and the associated hardware. However, the OCSWeb library provides a framework for generating basic GUI controls and displays, along with examples of how to attach handlers to dispatch Agent requests and process returned information. The goal is that Agent developers not need to learn much JavaScript to produce a useful control panel. The basic library is somewhat limited, but does not preclude experienced JavaScript developers from creating more sophisticated, customized control panels should the need arise.

Plans are in development to support persistent configuration between sessions through some combination of a configuration server and HTTP cookies; this will permit views involving certain subsets of Agents to persist from session to session.

\section{LIVE MONITORING}

Remote live monitoring of telescope systems is critical to ensuring high observation efficiency. Our goal has been to provide a web interface for real time viewing of housekeeping systems that remote observers can use to assess the health of the observatory. These systems generally consist of slow data rate timestreams, on the order of several $\mathrm{Hz}$ sampling rates or slower. Higher data rate timestreams, such as the raw detector data, are more suitable for custom local interfaces. We searched for existing tools to solve this problem, and have chosen several open source tools.

\subsection{Grafana}

Grafanall is an open source observability web application used for visualization and analytics of time series data. It supports many time series database and relational database backends as "data sources". Once a data source is configured, and data are inserted into the database, Grafana can query the database and display the resulting data in a user's web browser dynamically. This is done on web pages called "dashboards", in plots called "panels". A screenshot showing a Grafana dashboard with several panels is shown in Figure 3.

Dashboards are persistent and can be loaded upon revisiting the web application. They can be configured to automatically refresh, and provide a modern web interface for timeseries plots. We have selected InfluxDB as our primary data source backend. All housekeeping data within ocsare published through the crossbar server via ocsFeeds. There are two primary subscribers to these feeds: the HK Aggregator and the InfluxDB Publisher. These both record separate copies of the HK data, to .g3 files and to the Influx Database, respectively.

\footnotetext{
"https://grafana.com/
} 


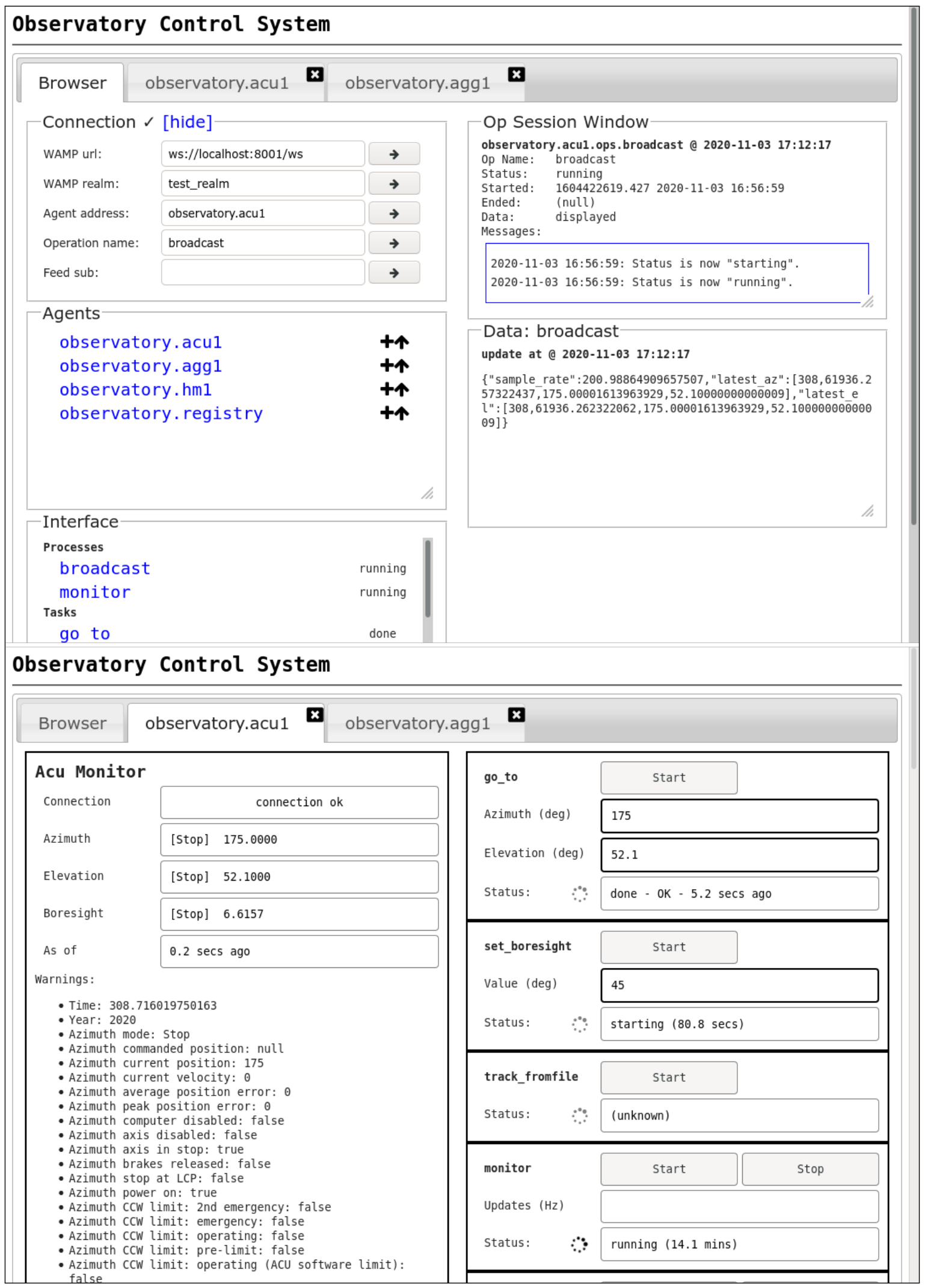

Figure 2. OCSWeb main page (top) and special control panel for the telescope platform control Agent (bottom). 


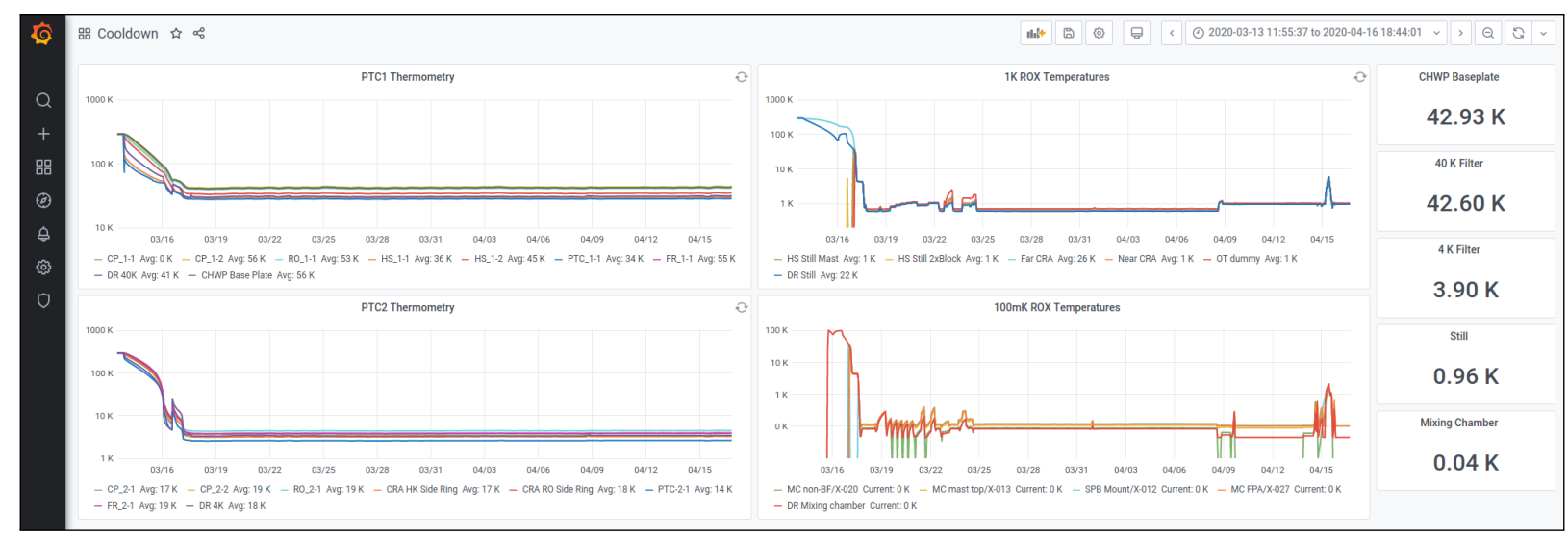

Figure 3. A screenshot of the Grafana live monitor showing cryogenic thermometry during a cooldown of the SAT1 receiver at UCSD. The y-axis in all plots is temperature in Kelvin and the $\mathrm{x}$-axis is time, showing approximately a month of time during which the cryostat was cooled and several tests were run.

\subsection{InfluxDB}

InfluxDB, a time series database developed by InfluxData,** is a popular backend data sources for Grafana, and is designed for fast access to large volumes of time series data. Hardware running InfluxDB must be sized appropriately, depending on the number of writes per second and the number of queries per second. We have found the performance suitable on moderate modern desktop computers typically found in labs for the slow housekeeping data within SO on shorter timescales, from one day to several months. Loading a significant amount of data can cause issues, so one must be aware of the queries configured within Grafana. We are exploring solutions to this: see Section 5.5.

\subsection{Log Aggregation with Loki}

Loki is a log aggregation system designed by Grafana Labs. Written by the same team that made Grafana, it integrates very well with the Grafana web interface. A logging driver for Docker publishes logs to the Loki service. Users can then follow logs or query for past logs directly within the Grafana web interface. Aggregation of logs external to Docker can be performed using a tool called promtail, also from Grafana Labs, however most of our requirements are met by the Docker logging driver so we we are not using it at this time. This may change in the future.

The interface allows for individual containers, or entire Compose services to be viewed. ocs logs simply print to stdout, which results in logging to Docker's configured logging driver. Logs remain visible via the "docker logs" command, while also being aggregated within Loki. This allows for easy viewing of logs in the event of an error somewhere within the ocssystem in a cohesive interface. The interface is shown in Figure 4.

\subsection{Detector Data}

The detector data volume is large enough that the HK live monitoring system cannot support the full detector data rate. The Pysmurf Monitor Agent has the ability to send a small subset of downsampled detector timestreams to the HK live monitoring system for basic checks. The full data rate detector timestreams can be monitored locally via a custom tool from the South Pole Telescope called lyrebird. ${ }^{\dagger \dagger}$ Lyrebird is being adapted for use on SO.

\footnotetext{
${ }^{* *}$ https://www.influxdata.com/

${ }^{\dagger \dagger}$ https://github.com/SouthPoleTelescope/lyrebird
} 


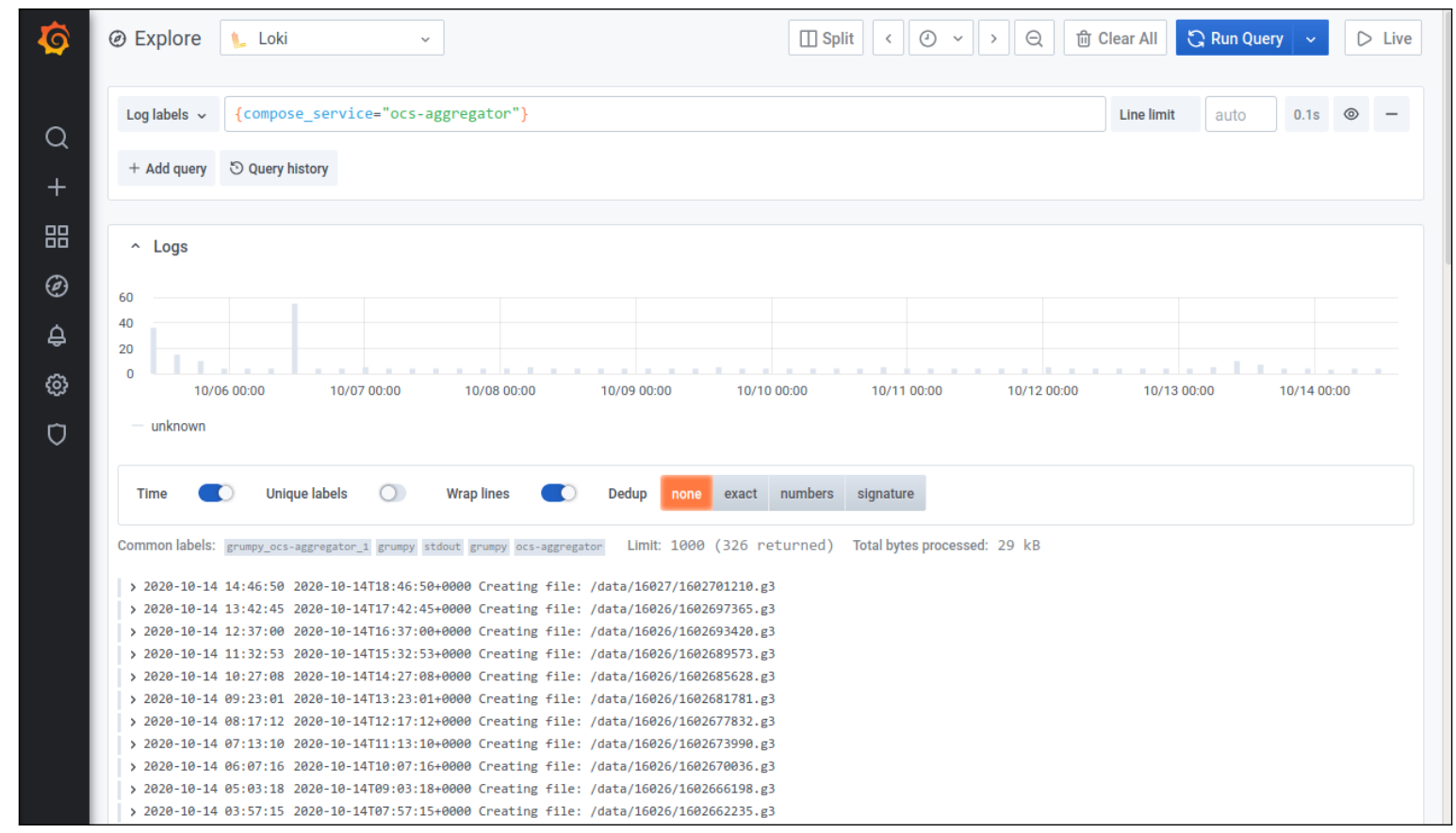

Figure 4. A screenshot of the Loki logs within Grafana showing the output of the ocsHK Aggregator agent. The top plot shows log frequency over time, and the bottom is a live feed of the log output. All Agents running within Docker containers can be configured to output logs to this interface.

\subsection{Challenges and Future Work}

Ideally we will retain all HK data in InfluxDB at the full rate throughout the lifetime of the experiment. However, as mentioned in Sec. 5.2, querying InfluxDB for long time ranges can cause issues, even with moderate data rates. Furthermore, given the limited resolution of any screen, requesting the full data rate for long time spans is inherently inefficient.

We are exploring solutions to this, which will likely involve returning lower sampling rates when viewing longer timescales. This will improve efficiency in queries to the database, improving performance. One likely solution is to use InfluxDB's "continuous queries" mechanism to downsample data and insert it into another database within InfluxDB. This could be done at various resolutions, optimized for different length timescales. Dashboards could then be configured to select the correct database depending on the users needs. Research remains to be done on this problem and will be ongoing.

\section{DEPLOYMENT}

ocs has been designed as a modular system. Except where Agents must communicate with specific hardware over dedicated connections or protocols, Agents can be run anywhere on the network. The use of Docker in the deployment of an ocs network further facilitates moving Agents to wherever computational resources are available. Dependencies for a given Agent are bundled within the pre-built Docker images, reducing the cost of moving the node an Agent is running on. This flexibility allows for many possible configurations. In this section we present a simple, single-node, deployment example, representative of what might be seen in a test lab. We then discuss the deployment at the University of California, San Diego (UCSD), which is close to what will be used on a single SAT. Lastly, we discuss the planned site layout for SO.

\subsection{Simple Deployment Example}

The simplest ocs deployment is on a single computer running all components needed for ocs: the crossbar server, any ocs Agents, and supporting monitoring infrastructure such as Grafana, a web server, and Loki for 
log aggregation (described in more detail in Sec. 5.3). Hardware with dedicated connections, such as USB connections, connect directly to this machine. Any networked devices that ocs sends commands to or receives data from must, of course, be on the same network as this machine. Figure 5 shows a diagram of this simple layout. ${ }^{\text {主 }}$

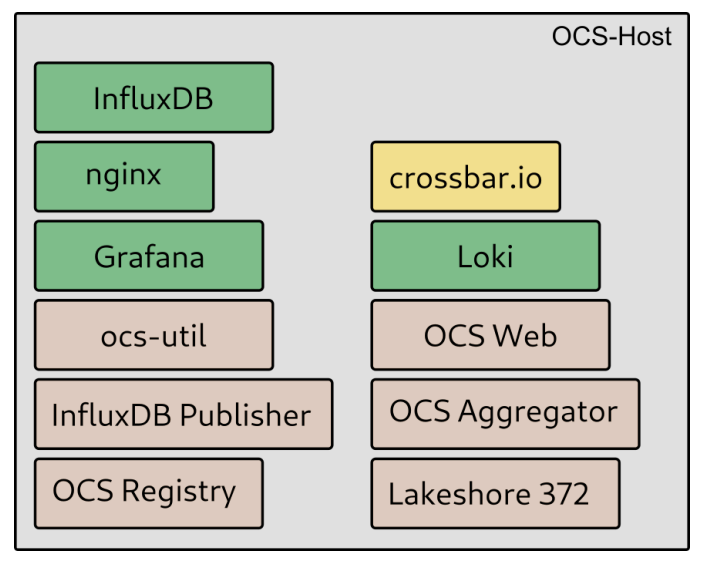

Figure 5. A diagram of a simple ocs network. A single node, "OCS-Host", runs all containers, represented by the colored boxes within the node. Connections to containers within the node are assumed. Users interact via external ocs Clients (not pictured here), or via OCSWeb and Grafana, served over the NGINX web server.

A modest desktop machine is capable of running this system. Most systems like this within SO are equipped with a quad-core CPU from the last 5-7 years and 12-16 GB of RAM. The example shown here could be used for readout and monitoring of a cryostat in the lab, with temperature control, hardware permitting. In this setting, data rates are typically low: $10 \mathrm{~Hz}$ or less per Agent.

\subsection{Test Lab Deployment and Development}

Test institution configurations will often span multiple nodes and operating systems, with network configurations that depend on many factors such as which agents are required to run and network restrictions imposed by the lab or university. In most cases there is a primary storage node that runs the core set of ocs agents and support containers, including the HK Aggregator and InfluxDB Publisher agents, the registry, the database containers and the Grafana server. Other nodes that are required to manage specific pieces of hardware such as the Bluefors DR or the SMuRF system are connected on a private subnet.

The most extensive ocs network in use is located at UCSD to test the integration of the SAT1. This system is running over 22 agents across five different nodes to control and monitor the SMuRF system, a cryogenic continuously rotating half wave plate (CHWP), Lakeshore devices, power supplies, the Bluefors DR, and other pieces of hardware. The network setup is shown in Figure 6.

\subsection{Planned Site Layout Deployment}

Once SO is fully deployed, the ocs network on-site will be more than four times as large as the ocs network discussed in Section 6.2. Each telescope will have an independent ocs network, meaning each will have its own crossbar server, HK Aggregator, InfluxDB Publisher, and collection of many other accompanying Agents. Most of these Agents will run within containers on a single node (one per telescope). Figure 7 shows a diagram of the planned site network.

Each SAT will have three specialized computers on the telescope platform, one for the cryogenic half-wave plate, one for the Bluefors DR, and one for the Lakeshore 240s. These will each run agents relevant to the hardware they couple to. Lastly, there are the SMuRF servers, which run the Agents and SMuRF Client

\footnotetext{
${ }_{\ddagger}^{\ddagger}$ An example close to this configuration is given in the "Quickstart" section of the ocs documentation.
} 


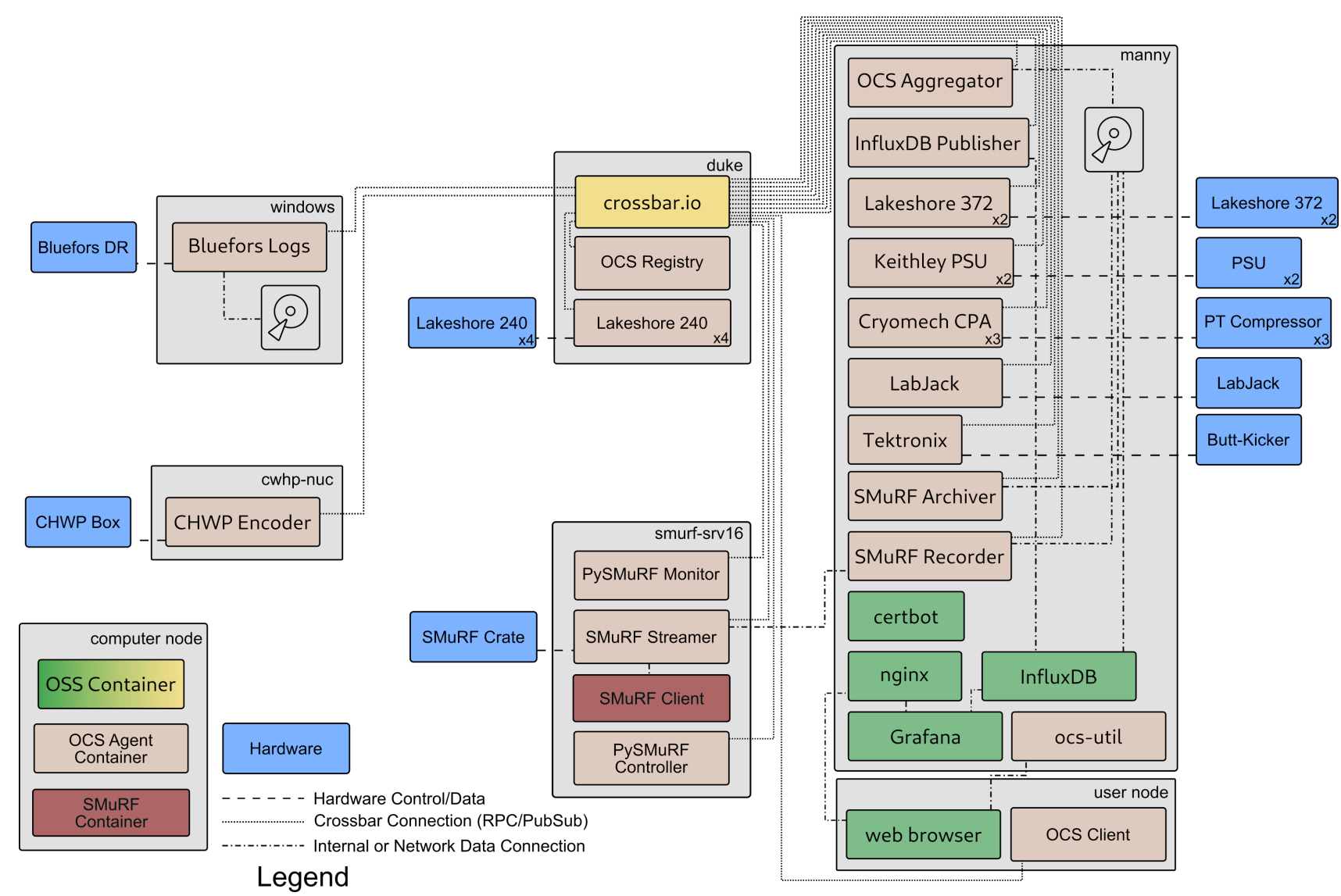

Figure 6. A diagram, similar to Figure 5 of the SAT1 development network at UCSD. Connections to the crossbar server are shown, as well as data connections, and connections to hardware. A legend describing the various connections and containers is shown in the bottom left. Open source software (OSS) containers are represented as either green or yellow boxes.

containers required to read out the detector arrays. There is one of these servers per SMuRF Crate, meaning there will be two per SAT and four for the LAT.

The LAT will have very similar Agents to those run on the SAT, though for some Agents there will be many more running instances of a given Agent, reflecting the larger overall size of the LAT. The LAT will not include a CHWP node or associated Agents.

In addition to the four separate ocs networks for each telescope, there will be one more network for site-wide hardware, such as the Meinberg M1000, used for site wide GPS synchronized timing, a weather monitor, to monitor the local weather, and several Agents related to power distribution at the site. These include UPS and iBootbar Agents, for battery backup of site computing infrastructure and remote power outlet control, and an Agent for interfacing with the on-site diesel generators.

Coordination of the ocs Agents distributed across these networks will be performed by the Observation Sequencer Agent described in Sec 3.3 where needed. All output data, whether from the ocs Aggregator or SMuRF Recorder, will end up on disk in a Redundant Array of Independent Disks (RAID) hosted on site. Data on this RAID will be available to local compute nodes which will perform near real time analysis of data for data quality assurance. Data will be further combined into units suitable for upload to our data transfer manager (a discussion of which is beyond the scope of this paper).

\subsubsection{Site Computing}

The ocs networks described in this section will run on a set powerful, multi-CPU, high RAM servers located in the control container on site, a temperature controlled and isolated environment. The SMuRF servers will 


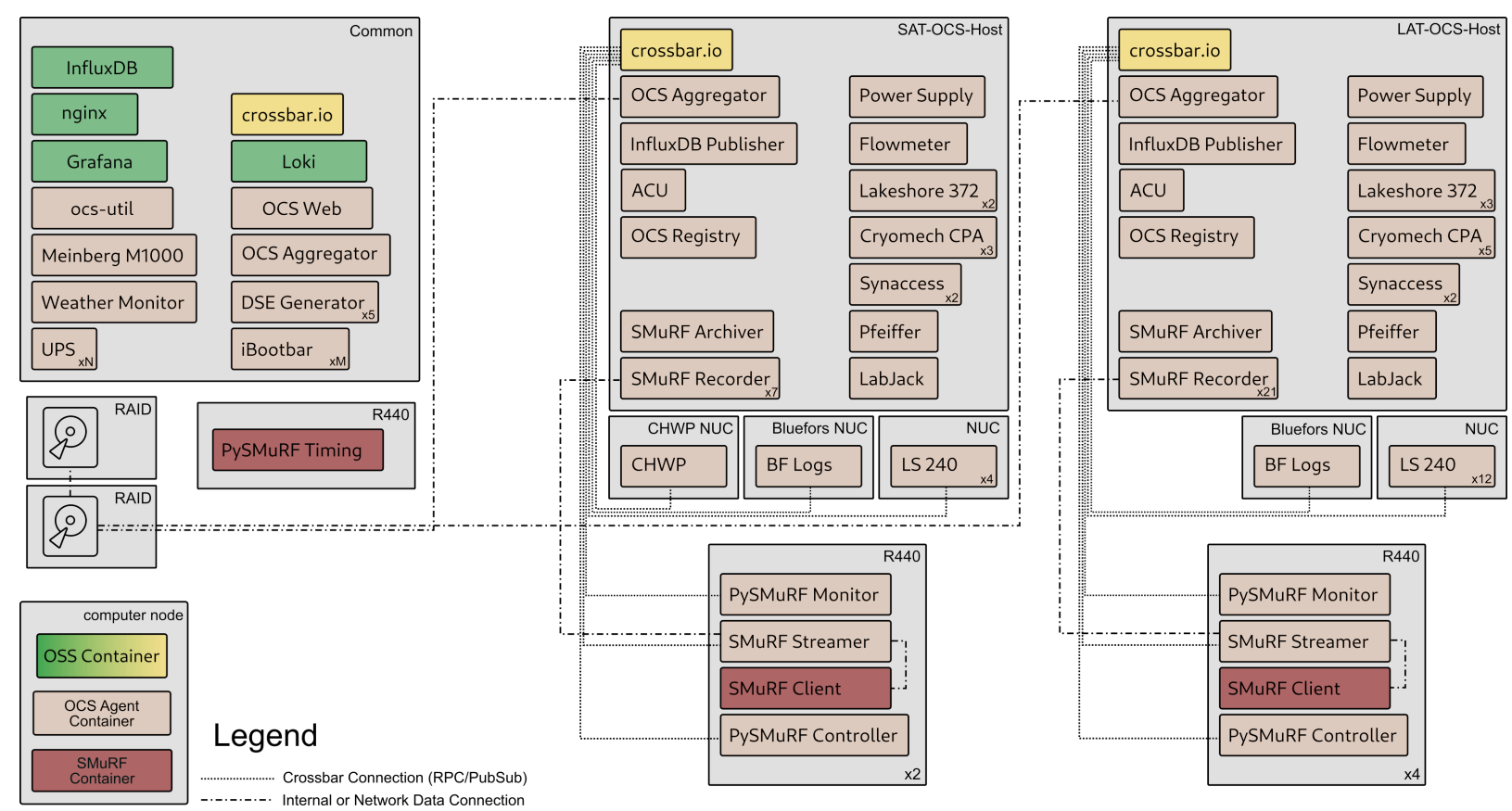

Figure 7. A diagram of the site ocs network. All nodes are connected via the on site network. Crossbar connections outside of a single node are shown. Connections within a node are assumed. One instance of an SAT ocs network is shown by the central group of nodes. At full deployment three of these will be present. Multiple copies of given Agents are shown with multipliers. The UPS and iBootbar Agent counts, $N$ and $M$, are still being determined.

also run in this location, and will run on Dell R440 servers. The dedicated NUC (short for Intel's "Next Unit of Computing", a small form factor PC) hardware on the telescope platforms are ruggedized Intel NUCs equipped with Intel Celeron processors.

The site will be equipped with a Meinberg M1000 timing system that synchronizes with GPS satellites and provides precision timing to the site network via the Precision Time Protocol (PTP) as well as inter-range instrumentation group (IRIG) timecodes and pulse per second (PPS) timing to the SMuRF master timing electronics. Each telescope platform will have a Meinberg SyncBox/N2X that accepts the PTP signal from the network and can be configured to output a variety of timing signals on three different BNC outputs. These will provide any precision timing signal required nearby the telescopes, such as for the CHWPs. The site networking equipment will all be PTP compatible, and appropriately configured to distribute the PTP timing signal within $\pm 100 \mathrm{~ns}$.

\section{SUMMARY}

We have presented an overview of the Observatory Control System for the Simons Observatory. The design and implementation of this distributed control system is modular and will scale up as we deploy the observatory and beyond. The use of open source tools, such as crossbar, Grafana, InfluxDB, and Docker, has allowed us to focus on the core ocs functionality while delivering a powerful monitoring interface, and convenient deployment options. The functionality provided by ocs is a critical aspect of the observatory and will enable the science goals outlined in our forecast paper. ${ }^{2}$

ocs is already integrated into the activities of SO through its use in labs doing hardware development and assembly. These preliminary deployments of ocs are not only contributing to the ongoing deployment of the experiment but have also been invaluable for debugging and developing our software. This process has been assisted by the use of Docker and by the automated testing and building of Docker images in the CI pipelines. The extensive documentation has enabled users at these labs to develop their own Agents and extend the functionality of ocs. ocs, and the SO specific Agents in socs, are both developed as open source software 
and are permissively licensed under the BSD 2-Clause license. The software is available on GitHub, and we encourage the community to try examples detailed within the documentation, to consider using ocs for their control software, and to contribute to its development.

\section{APPENDIX A. ACRONYMS}

Table 2 shows a list of all acronyms used in this paper.

\section{ACKNOWLEDGMENTS}

This work was funded by the Simons Foundation (Award \#457687). ZX is supported by the Gordon and Betty Moore Foundation. We would like to thank the communities behind the multiple open source packages in use with ocs.

\section{REFERENCES}

[1] Henderson, S. W., Ahmed, Z., Austermann, J., Becker, D., Bennett, D. A., Brown, D., Chaudhuri, S., Cho, H.-M. S., D’Ewart, J. M., Dober, B., Duff, S. M., Dusatko, J. E., Fatigoni, S., Frisch, J. C., Gard, J. D., Halpern, M., Hilton, G. C., Hubmayr, J., Irwin, K. D., Karpel, E. D., Kernasovskiy, S. S., Kuenstner, S. E., Kuo, C.-L., Li, D., Mates, J. A. B., Reintsema, C. D., Smith, S. R., Ullom, J., Vale, L. R., Winkle, D. D. V., Vissers, M., and Yu, C., "Highly-multiplexed microwave SQUID readout using the SLAC Microresonator Radio Frequency (SMuRF) electronics for future CMB and sub-millimeter surveys," Proc. SPIE 10708, 170 - 185 (2018).

[2] Ade, P., Aguirre, J., Ahmed, Z., Aiola, S., Ali, A., Alonso, D., Alvarez, M. A., Arnold, K., Ashton, P., Austermann, J., Awan, H., Baccigalupi, C., Baildon, T., Barron, D., Battaglia, N., Battye, R., Baxter, E., Bazarko, A., Beall, J. A., Bean, R., Beck, D., Beckman, S., Beringue, B., Bianchini, F., Boada, S., Boettger, D., Bond, J. R., Borrill, J., Brown, M. L., Bruno, S. M., Bryan, S., Calabrese, E., Calafut, V., Calisse, P., Carron, J., Challinor, A., Chesmore, G., Chinone, Y., Chluba, J., Cho, H.-M. S., Choi, S., Coppi, G., Cothard, N. F., Coughlin, K., Crichton, D., Crowley, K. D., Crowley, K. T., Cukierman, A., D'Ewart, J. M., Dünner, R., de Haan, T., Devlin, M., Dicker, S., Didier, J., Dobbs, M., Dober, B., Duell, C. J., Duff, S., Duivenvoorden, A., Dunkley, J., Dusatko, J., Errard, J., Fabbian, G., Feeney, S., Ferraro, S., Fluxà, P., Freese, K., Frisch, J. C., Frolov, A., Fuller, G., Fuzia, B., Galitzki, N., Gallardo, P. A., Tomas Galvez Ghersi, J., Gao, J., Gawiser, E., Gerbino, M., Gluscevic, V., Goeckner-Wald, N., Golec, J., Gordon, S., Gralla, M., Green, D., Grigorian, A., Groh, J., Groppi, C., Guan, Y., Gudmundsson, J. E., Han, D., Hargrave, P., Hasegawa, M., Hasselfield, M., Hattori, M., Haynes, V., Hazumi, M., He, Y., Healy, E., Henderson, S. W., Hervias-Caimapo, C., Hill, C. A., Hill, J. C., Hilton, G., Hilton, M., Hincks, A. D., Hinshaw, G., Hložek, R., Ho, S., Ho, S.-P. P., Howe, L., Huang, Z., Hubmayr, J., Huffenberger, K., Hughes, J. P., Ijjas, A., Ikape, M., Irwin, K., Jaffe, A. H., Jain, B., Jeong, O., Kaneko, D., Karpel, E. D., Katayama, N., Keating, B., Kernasovskiy, S. S., Keskitalo, R., Kisner, T., Kiuchi, K., Klein, J., Knowles, K., Koopman, B., Kosowsky, A., Krachmalnicoff, N., Kuenstner, S. E., Kuo, C.-L., Kusaka, A., Lashner, J., Lee, A., Lee, E., Leon, D., Leung, J. S. Y., Lewis, A., Li, Y., Li, Z., Limon, M., Linder, E., Lopez-Caraballo, C., Louis, T., Lowry, L., Lungu, M., Madhavacheril, M., Mak, D., Maldonado, F., Mani, H., Mates, B., Matsuda, F., Maurin, L., Mauskopf, P., May, A., McCallum, N., McKenney, C., McMahon, J., Meerburg, P. D., Meyers, J., Miller, A., Mirmelstein, M., Moodley, K., Munchmeyer, M., Munson, C., Naess, S., Nati, F., Navaroli, M., Newburgh, L., Nguyen, H. N., Niemack, M., Nishino, H., Orlowski-Scherer, J., Page, L., Partridge, B., Peloton, J., Perrotta, F., Piccirillo, L., Pisano, G., Poletti, D., Puddu, R., Puglisi, G., Raum, C., Reichardt, C. L., Remazeilles, M., Rephaeli, Y., Riechers, D., Rojas, F., Roy, A., Sadeh, S., Sakurai, Y., Salatino, M., Sathyanarayana Rao, M., Schaan, E., Schmittfull, M., Sehgal, N., Seibert, J., Seljak, U., Sherwin, B., Shimon, M., Sierra, C., Sievers, J., Sikhosana, P., Silva-Feaver, M., Simon, S. M., Sinclair, A., Siritanasak, P., Smith, K., Smith, S. R., Spergel, D., Staggs, S. T., Stein, G., Stevens, J. R., Stompor, R., Suzuki, A., Tajima, O., Takakura, S., Teply, G., Thomas, D. B., Thorne, B., Thornton, R., Trac, H., Tsai, C., Tucker, C., Ullom, J., Vagnozzi, S., van Engelen, A., Van Lanen, J., Van Winkle, D. D., Vavagiakis, E. M., Vergès, C., Vissers, M., Wagoner, K., Walker, S., Ward, J., Westbrook, B., Whitehorn, N., Williams, J., Williams, J., Wollack, E. J., Xu, Z., Yu, B., Yu, C., Zago, F., Zhang, H., Zhu, N., and Simons Observatory 
Collaboration, "The Simons Observatory: science goals and forecasts," J. Cosmology Astropart. Phys. 2019, 056 (Feb. 2019).

[3] Lee, A., Abitbol, M. H., Adachi, S., Ade, P., Aguirre, J., Ahmed, Z., Aiola, S., Ali, A., Alonso, D., Alvarez, M. A., Arnold, K., Ashton, P., Atkins, Z., Austermann, J., Awan, H., Baccigalupi, C., Baildon, T., Baleato Lizancos, A., Barron, D., Battaglia, N., Battye, R., Baxter, E., Bazarko, A., Beall, J. A., Bean, R., Beck, D., Beckman, S., Beringue, B., Bhandarkar, T., Bhimani, S., Bianchini, F., Boada, S., Boettger, D., Bolliet, B., Bond, J. R., Borrill, J., Brown, M. L., Bruno, S. M., Bryan, S., Calabrese, E., Calafut, V., Calisse, P., Carron, J., Carl, F. M., Cayuso, J., Challinor, A., Chesmore, G., Chinone, Y., Chluba, J., Cho, H.-M. S., Choi, S., Clark, S., Clarke, P., Contaldi, C., Coppi, G., Cothard, N. F., Coughlin, K., Coulton, W., Crichton, D., Crowley, K. D., Crowley, K. T., Cukierman, A., D'Ewart, J. M., Dünner, R., de Haan, T., Devlin, M., Dicker, S., Dober, B., Duell, C. J., Duff, S., Duivenvoorden, A., Dunkley, J., El Bouhargani, H., Errard, J., Fabbian, G., Feeney, S., Fergusson, J., Ferraro, S., Fluxa, P., Freese, K., Frisch, J. C., Frolov, A., Fuller, G., Galitzki, N., Gallardo, P. A., Galvez Ghersi, J. T., Gao, J., Gawiser, E., Gerbino, M., Gluscevic, V., Goeckner-Wald, N., Golec, J., Gordon, S., Gralla, M., Green, D., Grigorian, A., Groh, J., Groppi, C., Guan, Y., Gudmundsson, J. E., Halpern, M., Han, D., Hargrave, P., Harrington, K., Hasegawa, M., Hasselfield, M., Hattori, M., Haynes, V., Hazumi, M., Healy, E., Henderson, S. W., Hensley, B., Hervias-Caimapo, C., Hill, C. A., Hill, J. C., Hilton, G., Hilton, M., Hincks, A. D., Hinshaw, G., Hložek, R., Ho, S., Ho, S.-P. P., Hoang, T. D., Hoh, J., Hotinli, S. C., Huang, Z., Hubmayr, J., Huffenberger, K., Hughes, J. P., Ijjas, A., Ikape, M., Irwin, K., Jaffe, A. H., Jain, B., Jeong, O., Johnson, M., Kaneko, D., Karpel, E. D., Katayama, N., Keating, B., Keskitalo, R., Kisner, T., Kiuchi, K., Klein, J., Knowles, K., Kofman, A., Koopman, B., Kosowsky, A., Krachmalnicoff, N., Kusaka, A., La Plante, P., Lashner, J., Lee, A., Lee, E., Lewis, A., Li, Y., Li, Z., Limon, M., Linder, E., Liu, J., Lopez-Caraballo, C., Louis, T., Lungu, M., Madhavacheril, M., Mak, D., Maldonado, F., Mani, H., Mates, B., Matsuda, F., Maurin, L., Mauskopf, P., May, A., McCallum, N., McCarrick, H., McKenney, C., McMahon, J., Meerburg, P. D., Mertens, J., Meyers, J., Miller, A., Mirmelstein, M., Moodley, K., Moore, J., Munchmeyer, M., Munson, C., Murata, M., Naess, S., Namikawa, T., Nati, F., Navaroli, M., Newburgh, L., Nguyen, H. N., Nicola, A., Niemack, M., Nishino, H., Nishinomiya, Y., Orlowski-Scherer, J., Pagano, L., Partridge, B., Perrotta, F., Phakathi, P., Piccirillo, L., Pierpaoli, E., Pisano, G., Poletti, D., Puddu, R., Puglisi, G., Raum, C., Reichardt, C. L., Remazeilles, M., Rephaeli, Y., Riechers, D., Rojas, F., Rotti, A., Roy, A., Sadeh, S., Sakurai, Y., Salatino, M., Sathyanarayana Rao, M., Saunders, L., Schaan, E., Schmittfull, M., Sehgal, N., Seibert, J., Seljak, U., Shellard, P., Sherwin, B., Shimon, M., Sierra, C., Sievers, J., Sifon, C., Sikhosana, P., Silva-Feaver, M., Simon, S. M., Sinclair, A., Smith, K., Sohn, W., Sonka, R., Spergel, D., Spisak, J., Staggs, S. T., Stein, G., Stevens, J. R., Stompor, R., Suzuki, A., Tajima, O., Takakura, S., Teply, G., Thomas, D. B., Thorne, B., Thornton, R., Trac, H., Treu, J., Tsai, C., Tucker, C., Ullom, J., Vagnozzi, S., van Engelen, A., Van Lanen, J., Van Winkle, D. D., Vavagiakis, E. M., Vergès, C., Vissers, M., Wagoner, K., Walker, S., Wang, Y., Ward, J., Westbrook, B., Whitehorn, N., Williams, J., Williams, J., Wollack, E., Xu, Z., Yasini, S., Young, E., Yu, B., Yu, C., Zago, F., Zannoni, M., Zhang, H., Zheng, K., Zhu, N., and Zonca, A., "The Simons Observatory," in [Bulletin of the American Astronomical Society], 51, 147 (Sept. 2019).

[4] Story, K., Leitch, E., Ade, P., Aird, K. A., Austermann, J. E., Beall, J. A., Becker, D., Bender, A. N., Benson, B. A., Bleem, L. E., Britton, J., Carlstrom, J. E., Chang, C. L., Chiang, H. C., Cho, H.-M., Crawford, T. M., Crites, A. T., Datesman, A., de Haan, T., Dobbs, M. A., Everett, W., Ewall-Wice, A., George, E. M., Halverson, N. W., Harrington, N., Henning, J. W., Hilton, G. C., Holzapfel, W. L., Hoover, S., Huang, N., Hubmayr, J., Irwin, K. D., Karfunkle, M., Keisler, R., Kennedy, J., Lee, A. T., Li, D., Lueker, M., Marrone, D. P., McMahon, J. J., Mehl, J., Meyer, S. S., Montgomery, J., Montroy, T. E., Nagy, J., Natoli, T., Nibarger, J. P., Niemack, M. D., Novosad, V., Padin, S., Pryke, C., Reichardt, C. L., Ruhl, J. E., Saliwanchik, B. R., Sayre, J. T., Schaffer, K. K., Shirokoff, E., Smecher, G., Stalder, B., Tucker, C., Vanderlinde, K., Vieira, J. D., Wang, G., Williamson, R., Yefremenko, V., Yoon, K. W., and Young, E., "South Pole Telescope software systems: control, monitoring, and data acquisition," Proc. SPIE 8451, 286 - 296 (2012).

[5] BICEP2 Collaboration, Ade, P. A. R., Aikin, R. W., Amiri, M., Barkats, D., Benton, S. J., Bischoff, C. A., Bock, J. J., Brevik, J. A., Buder, I., Bullock, E., Davis, G., Day, P. K., Dowell, C. D., Duband, L., Filippini, J. P., Fliescher, S., Golwala, S. R., Halpern, M., Hasselfield, M., Hildebrandt, S. R., Hilton, G. C., Irwin, 
K. D., Karkare, K. S., Kaufman, J. P., Keating, B. G., Kernasovskiy, S. A., Kovac, J. M., Kuo, C. L., Leitch, E. M., Llombart, N., Lueker, M., Netterfield, C. B., Nguyen, H. T., O'Brient, R., Ogburn, R. W., I., Orlando, A., Pryke, C., Reintsema, C. D., Richter, S., Schwarz, R., Sheehy, C. D., Staniszewski, Z. K., Story, K. T., Sudiwala, R. V., Teply, G. P., Tolan, J. E., Turner, A. D., Vieregg, A. G., Wilson, P., Wong, C. L., and Yoon, K. W., "BICEP2. II. Experiment and three-year Data Set," ApJ 792, 62 (Sept. 2014).

[6] Switzer, E. R., Allen, C., Amiri, M., Appel, J. W., Battistelli, E. S., Burger, B., Chervenak, J. A., Dahlen, A. J., Das, S., Devlin, M. J., Dicker, S. R., Doriese, W. B., Dünner, R., Essinger-Hileman, T., Gao, X., Halpern, M., Hasselfield, M., Hilton, G. C., Hincks, A. D., Irwin, K. D., Knotek, S., Fisher, R. P., Fowler, J. W., Jarosik, N., Kaul, M., Klein, J., Lau, J. M., Limon, M., Lupton, R. H., Marriage, T. A., Martocci, K. L., Moseley, S. H., Netterfield, C. B., Niemack, M. D., Nolta, M. R., Page, L., Parker, L. P., Reid, B. A., Reintsema, C. D., Sederberg, A. J., Sievers, J. L., Spergel, D. N., Staggs, S. T., Stryzak, O. R., Swetz, D. S., Thornton, R. J., Wollack, E. J., and Zhao, Y., "Systems and control software for the Atacama Cosmology Telescope," in [Advanced Software and Control for Astronomy II], Bridger, A. and Radziwill, N. M., eds., 7019, 856 - 867, International Society for Optics and Photonics, SPIE (2008).

[7] Swetz, D. S., Ade, P. A. R., Amiri, M., Appel, J. W., Battistelli, E. S., Burger, B., Chervenak, J., Devlin, M. J., Dicker, S. R., Doriese, W. B., Dünner, R., Essinger-Hileman, T., Fisher, R. P., Fowler, J. W., Halpern, M., Hasselfield, M., Hilton, G. C., Hincks, A. D., Irwin, K. D., Jarosik, N., Kaul, M., Klein, J., Lau, J. M., Limon, M., Marriage, T. A., Marsden, D., Martocci, K., Mauskopf, P., Moseley, H., Netterfield, C. B., Niemack, M. D., Nolta, M. R., Page, L. A., Parker, L., Staggs, S. T., Stryzak, O., Switzer, E. R., Thornton, R., Tucker, C., Wollack, E., and Zhao, Y., "Overview of the Atacama Cosmology Telescope: Receiver, Instrumentation, and Telescope Systems," The Astrophysical Journal Supplement Series 194, 41 (June 2011).

[8] Thornton, R. J., Ade, P. A. R., Aiola, S., Angilè, F. E., Amiri, M., Beall, J. A., Becker, D. T., Cho, H.-M., Choi, S. K., Corlies, P., Coughlin, K. P., Datta, R., Devlin, M. J., Dicker, S. R., Dünner, R., Fowler, J. W., Fox, A. E., Gallardo, P. A., Gao, J., Grace, E., Halpern, M., Hasselfield, M., Henderson, S. W., Hilton, G. C., Hincks, A. D., Ho, S. P., Hubmayr, J., Irwin, K. D., Klein, J., Koopman, B., Li, D., Louis, T., Lungu, M., Maurin, L., McMahon, J., Munson, C. D., Naess, S., Nati, F., Newburgh, L., Nibarger, J., Niemack, M. D., Niraula, P., Nolta, M. R., Page, L. A., Pappas, C. G., Schillaci, A., Schmitt, B. L., Sehgal, N., Sievers, J. L., Simon, S. M., Staggs, S. T., Tucker, C., Uehara, M., Lanen, J. v., Ward, J. T., and Wollack, E. J., "The Atacama Cosmology Telescope: The Polarization-Sensitive ACTPol Instrument," The Astrophysical Journal Supplement Series 227, 21 (Dec. 2016).

[9] Koopman, B. J., Detector Development and Polarization Analyses for the Atacama Cosmology Telescope, $\mathrm{PhD}$ thesis, Cornell U. (2018).

[10] Harrington, N., Constraints on Short Lived Signals at 150 GHz, PhD thesis, University of California, Berkeley (2018). https://escholarship.org/uc/item/7jv1d54w.

[11] Kernasovskiy, S. A., Kuenstner, S. E., Karpel, E., Ahmed, Z., Van Winkle, D. D., Smith, S., Dusatko, J., Frisch, J. C., Chaudhuri, S., Cho, H. M., Dober, B. J., Henderson, S. W., Hilton, G. C., Hubmayr, J., Irwin, K. D., Kuo, C. L., Li, D., Mates, J. A. B., Nasr, M., Tantawi, S., Ullom, J., Vale, L., and Young, B., "SLAC Microresonator Radio Frequency (SMuRF) Electronics for Read Out of Frequency-DivisionMultiplexed Cryogenic Sensors," arXiv:1805.08363 [astro-ph] (May 2018). Comment: 7 pages, 5 figures, Submitted to the Journal of Low Temperature Physics (Proceedings of the 17th International Workshop on Low Temperature Detectors). 


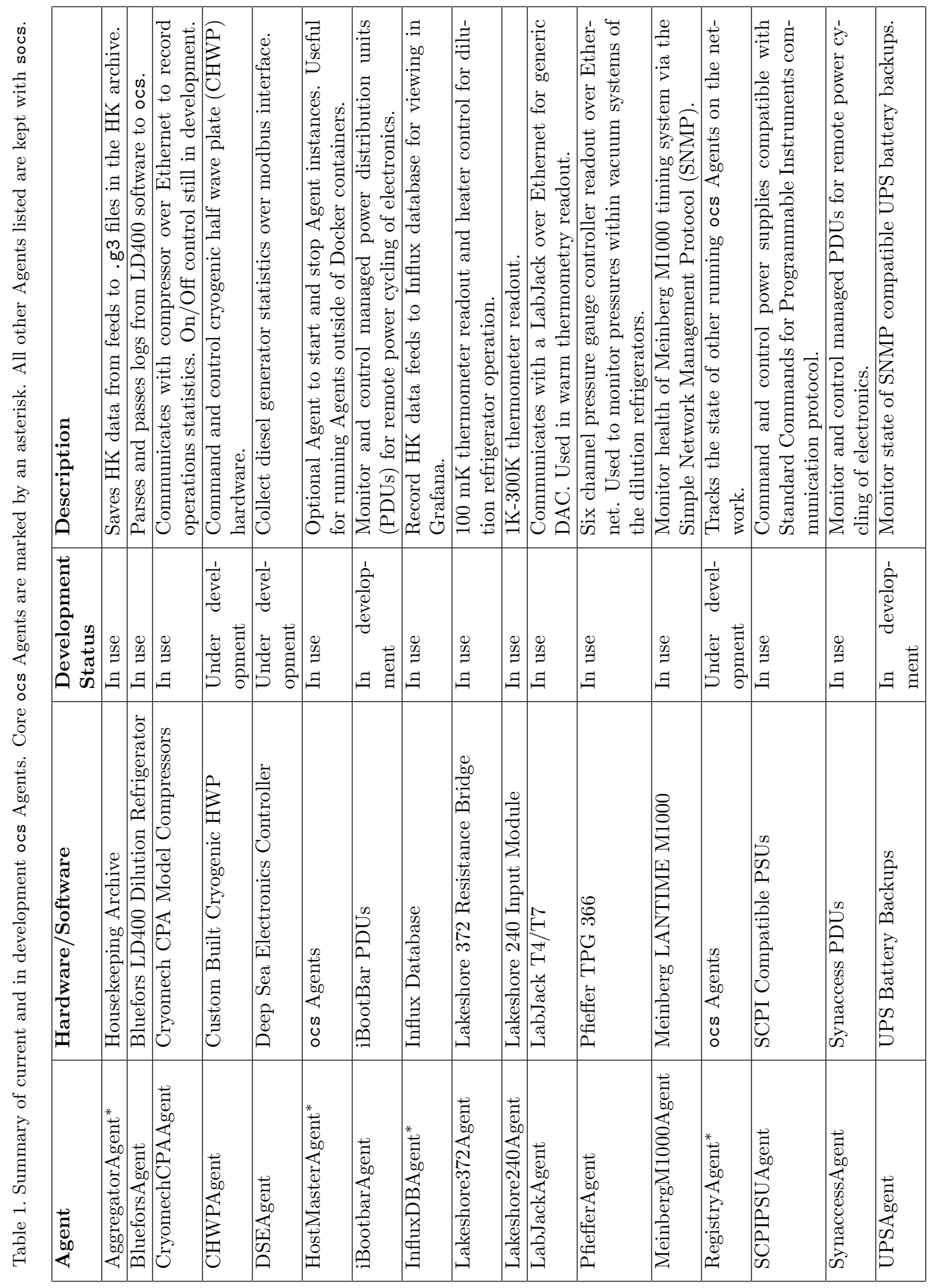


Table 2. Acronyms.

\begin{tabular}{|c|c|}
\hline Acronym & Meaning or Explanation \\
\hline ACT & The Atacama Cosmology Telescope \\
\hline $\mathrm{ACU}$ & Antenna Control Unit \\
\hline API & Application Programming Interface \\
\hline BICEP & Background Imaging of Cosmic Extragalactic Polarization Telescope \\
\hline $\mathrm{BNC}$ & Bayonet Neill-Concelman, a common coaxial connector type \\
\hline CI & Continuous Integration \\
\hline $\mathrm{CPU}$ & Central processing unit \\
\hline CSS & Cascading Style Sheets \\
\hline CWHP & Cryogenic Half-wave Plate \\
\hline DR & Dilution Refrigerator \\
\hline EPICS & Experimental Physics and Industrial Control System \\
\hline FPGA & Field-programmable gate array \\
\hline .93 & Short term for referring to spt3g_software \\
\hline GCP & General Control Program, control software used in past CMB experiments \\
\hline GPS & Global Positioning System \\
\hline GUI & Graphical user interface \\
\hline HK & Housekeeping data (i.e. non-detector data) \\
\hline HTML & Hypertext Markup Language \\
\hline $\operatorname{HTTP}(\mathrm{S})$ & Hypertext Transfer Protocol (Secure) \\
\hline IRIG & Inter-Range Instrumentation Group \\
\hline LAT & Large Aperture Telescope \\
\hline NUC & Intel's "Next Unit of Computing" \\
\hline $\mathrm{PC}$ & Personal computer \\
\hline PPS & Pulse per second \\
\hline PTP & Precision Time Protocol \\
\hline Pubsub & Publish/Subscribe \\
\hline ocs & Observatory Control System \\
\hline RAID & Redundant Array of Independent Disks \\
\hline RAM & Random-access memory \\
\hline $\mathrm{RPC}$ & Remote procedure call \\
\hline SAT & Small Aperture Telescope \\
\hline $\mathrm{SCF}$ & OCS Site Configuration File \\
\hline SLAC & SLAC National Accelerator Laboratory \\
\hline SMuRF & SLAC Microresonator Radio Frequency \\
\hline SPT & South Pole Telescope \\
\hline SO & Simons Observatory \\
\hline socs & Simons Observatory Control System \\
\hline SPT3G & The South Pole Telescope 3rd Generation, can also refer to the spt3g software package \\
\hline TCP & Transmission Control Protocol \\
\hline TES & Transition Edge Sensor \\
\hline UCSD & University of California, San Diego \\
\hline UDP & User Datagram Protocol \\
\hline$\mu \mathrm{MUX}$ & Microwave multiplexing \\
\hline UPS & Uninterruptible power supply \\
\hline USB & Universal Serial Bus \\
\hline WAMP & Web Application Messaging Protocol \\
\hline YAML & Yet Another Markup Language \\
\hline
\end{tabular}

